\title{
V018 RESERVOIR MONITORING - THE TOOLS AND THE LOOPS
}

\section{EXPANDED ABSTRACT:}

Reservoir characterization and reservoir monitoring is a scientific and mathematical discipline which defines input data needed to predict (by simulation) and confirm (by measurement flow through permeable media. This implies that cross-disciplinary integration has to be established.

The reservoir simulator in principle gives pressure changes and saturation changes in the spaces between the history-matched wells, based on a block model of the reservoir.

Fig.1 gives a summary of the tools and the loops envisioned for a successful implementation of reservoir monitoring.

In terms of measurements we restrict the following discussion to surface seismics and VSP/MSP - data, either vertical component only or up to three components. The conventional view today here is that repeated measurements (in terms of P-wave data!) are required to map changes in the pore-space of the reservoir resulting from ongoing production of Hydrocarbons. The initial data set is called the BASE-LINE-SURVEY (which of course could be and should be linked to already existing seismic data sets from previous $2 \mathrm{D}$-surveys), which then is followed by REPEATED SURVEYS (which will cover only a subset of the initial area for obvious reasons). The acquisition-geometry of the surveys to be compared should be identical for reasons of multiples, $\mathrm{S} / \mathrm{N}$-ratio etc, which implies that the acquisition geometry of the repeated survey has to contain that of the base-line-survey. This means that we can modify the new acquisition geometry relative to the old one, provided that one can simulate by decimation the old geometry from the extended new one. From the base-line-survey normally the structural model in terms of velocity distribution and reflector geometry is derived by using the first two phases (out of three in total) of processing, i.e. TIME DOMAIN AND POST STACK DEPTH DOMAIN-processing.

Depending on the validity of the ZERO-OFFSET-(ZO)-CONDITION (i.e. does the DMO-stack represent a Zero-Offset data volume) , locally" the third phase in data processing, i.e. PRE STACK DEPTH DOMAIN may have to be applied in order to achieve the optimum, i.e. correct image of the data-volume. This final result (in terms of eg fault definitions) could be of utmost importance for local block refinement in the simulatormodel.

The next step is the SEQUENCE-STRATIGRAPHIC INTERPRETATION of the reservoir-interval (carbonates or siliciclastics) in question, resulting in e.g. SHELF TRENDS, CHANNEL DISTRIBUTIONS, etc and finally in the LITHO MODEL of the reservoir. The litho units are described by their acoustic impedances (AI), and well-data are used here for that purpose. In addition normally INVERSION FOR AI of the migrated data-volume (i.e. usually only a sub-volume containing the resẹrvoir-interval is used) is carried out as well and subsequently used by the interpreter.

In parallel „engineering equations" for the $\mathrm{P}$ - and (if available) the S-wave velocities are established, which typically have the following form:

$\mathrm{Vp}, \mathrm{s}=\mathrm{Vo}+(\partial \mathrm{V} / \partial \phi) \cdot \phi+(\partial \mathrm{V} / \partial \mathrm{p}) \cdot \mathrm{p}$, where $\phi=$ porosity, $\mathrm{p}=$ calcite content for carbonates or clay content for sandstones

The established Litho Model then is confirmed/corrected by FORWARD MODELLING (acoustic/elastic), i.e. the seismic response of the Litho Model is checked against the actual measurement. This type of checking is called the VERIFICATION LOOP.

The next steo is to quantify the effects of the different pore-fills within the reservoir (See Fig.2) using the engineering equations in conjunction with the BIOT-GASSMANN-THEORY.

For this part one needs to know if the reservoir is above the bubble point line (i.e. fluids only) or not (fluids and gas). Clearly in the case of gas being present SHEAR WAVES (S/S) are needed in order to be able to quantify for gas saturation, i.e. the acoustic impedance for the $S / S$-wavefield is needed here.

The P/S-WAVEFIELD in the presence of gas is just an anomaly indicator like P/P-AVO.

An interesting additional aspect here is in case of FRACTURED RESERVOIRS , that the S/S-reflection coefficient (and the P/S-reflection coefficient as well) is sensitive to FRACTURE INTENSITY.

The final result of this exercise is a data-base containing velocities/densities/impedances along with the individual moduli/elastic constants for a range of lithologies/porosities/saturations, allowing to quantify for the effect of variing pore-fill, based on impedance changes due to pore fill-changes. 
It is worthwile to note that shear wave data $(S / S)$, since they allow a DIRECT QUANTIFICATION via the corresponding DENSITY CHANGE, in principle do not require repeated measurements! This is important e.g. in terms of SECONDARY RECOVERY, if one changes from water injection to WAG (= water-alternating-gas).

For P-data in principle for the fluid-case (i.e. above bubble point) we may think of quantification in terms of the fluid modulus $\mathrm{KF}$ as well by a single measurement, but have the problem of wetting/non wetting phases and drainage/imbibition. Therefore a repetition of surveys using P-waves at present seems to be unavoidable.

In addition the combination of $\mathrm{P}$ and $\mathrm{S}$ allows to map the $\mathrm{Vp} / \mathrm{Vs}$-ratio as well, which is a convenient lithology/ fracture/etc-indicator, i.e. in case of sandstones the ratio gives a Gamma Ray log and in case of carbonates the ratio gives a density log.

The splitting phenomenon for S-waves on the other hand at least is "very difficult" to be used in conjunction with surface-seismic data, but is very effective and stable, if we measure it at two different depth-levels (VSPaspect).

The last step then is to go for INVERSION FOR AI (usually L1-Norm spike deconvolution, followed by Born-inversion) of the base-line-survey and the repeated survey, and to map the IMPEDANCE CHANGES within the reservoir. These impedance-changes have to be compared with the result of the parallel reservoirsimulation, i.e. PRESSURE- AND SATURATION CHANGES (See Fig.1).

A local mismatch between these two sets then should result in a local correction of the simulator model and a rerun of the simulation.

A sensitivity analysis should preceed above loop in order to define the range of values, within which the QUANTIFICATION (i.e. the impedance change-to-saturation change transform) is reliable and applicable. At this stage a local zoom into the reservoir by using e.g. additional data acquisition (shear waves, VSP/MSP) is envisioned as a possible complementary optional step.

Finally the improved HC-recovery in terms of additional production should be quantified as well.

\section{REFERENCES:}

Wang,Z.,Nur,A.(1980): Dispersion analysis of acoustic velocities in rocks: J.Acoust.Soc.Am.,87(6),June 1980)

Dake,.L.P.(1978): Fundamentals of reservoir engineering: Elsevier, Amsterdam

Li,X-Y.,Kühnel,T.,Macbeth,C.(1996): Converted wave AVO and ist implications: 58th EAGE,Amsterdam, paper M046

Marschall, R.(1996):Reservoir Seismics: paper presented at Techn.University Clausthal-Zellerfeld, Colloquium, June 24,1996.

Gawith,D.E.,Gutteridge,P.A.(1996): Seismic validation of reservoir simulation using a Shared Earth Model: Petr.Geosc.,Vol.2,p.97-103

Nieuwland,F.,Marschall,R.,Papaterpos,M.,Sharp,D.(1994): An example of the use of shear waves in seismic exploration: Journ.Seism.Expl.,Vol.3,p.5-20

Krief,M.,Garat,J.,Stellingwerth,J.,Ventre,J.(1990): A petrophysical interpretation using the velocities of $P$ and S waves (Full wave form sonic): The Log Analyst,Nov.-Dec.1990,p.355-369

Fischer,K.C.,Möller,U.,Marschall,R.(1994): Development of an exploration concept for the Shuaiba Formation (Thamama group) using seismic sequence and facies analysis in combination with forward modelling: GEO’94, Vol.I (Ed.: M. Al-Husseini), p.377-386, Guif Petro Link, Bahrain. 


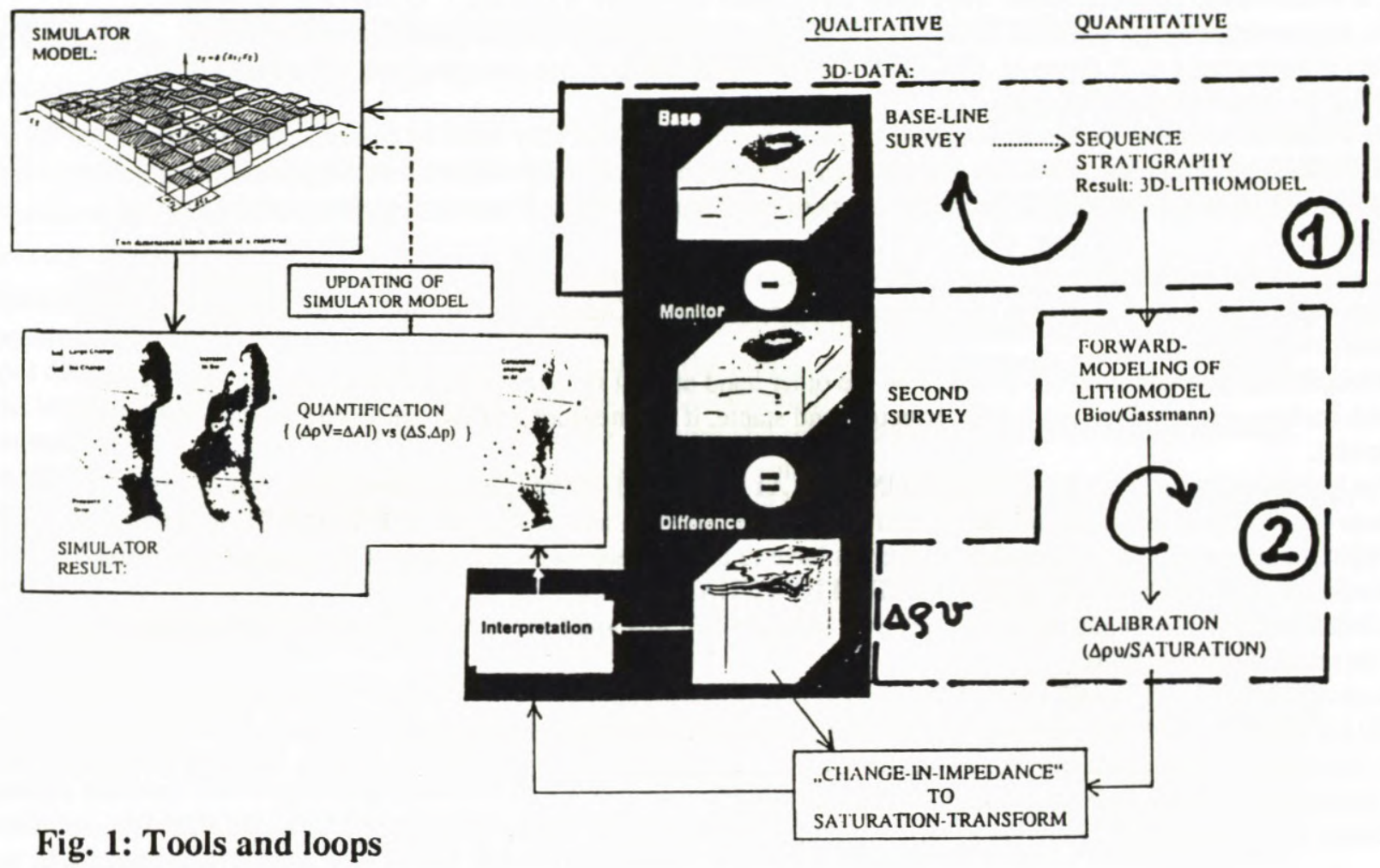

SO WE MAY EXPECT THE FOLLOWING

„REGIONS OF USEFULNESS“ FOR P-AND S-WAVES:

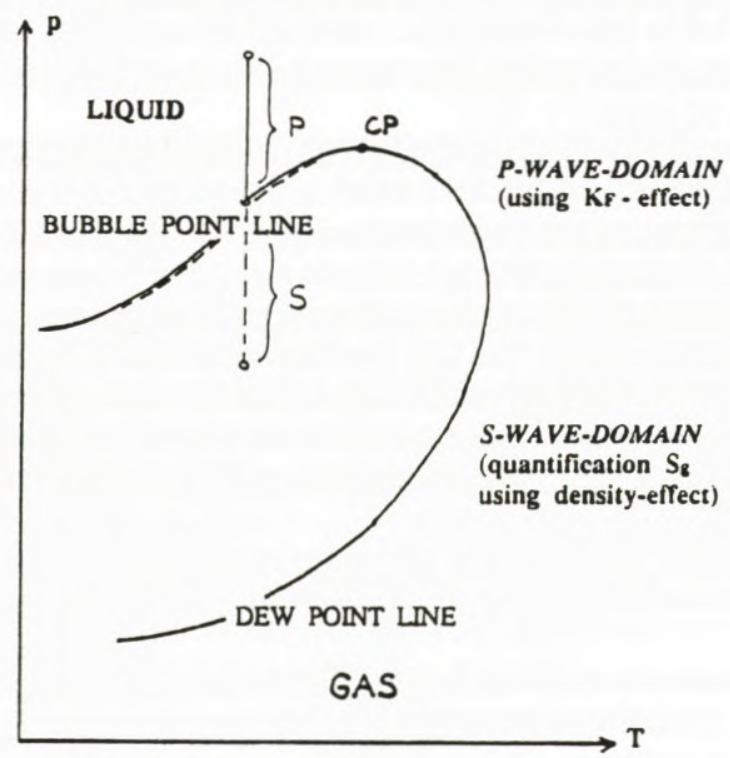

Fig.2: Reservoir above/below

Bubble Point 
